\title{
Resveratrol regulates neuro-inflammation and induces adaptive immunity in Alzheimer's disease
}

\author{
Charbel Moussa ${ }^{1 *} \mathbb{D}$, Michaeline Hebron', Xu Huang ${ }^{1}$, Jaeil Ahn², Robert A. Rissman², Paul S. Aisen ${ }^{4}$
} and R. Scott Turner ${ }^{5}$

\begin{abstract}
Background: Treatment of mild-moderate Alzheimer's disease (AD) subjects ( $N=119)$ for 52 weeks with the SIRT1 activator resveratrol (up to $1 \mathrm{~g}$ by mouth twice daily) attenuates progressive declines in CSF A 340 levels and activities of daily living (ADL) scores.

Methods: For this retrospective study, we examined banked CSF and plasma samples from a subset of AD subjects with CSF A $42<600 \mathrm{ng} / \mathrm{ml}$ (biomarker-confirmed AD) at baseline ( $N=19$ resveratrol-treated and $N=19$ placebo-treated). We utilized multiplex Xmap technology to measure markers of neurodegenerative disease and metalloproteinases (MMPs) in parallel in CSF and plasma samples.

Results: Compared to the placebo-treated group, at 52 weeks, resveratrol markedly reduced CSF MMP9 and increased macrophage-derived chemokine (MDC), interleukin (IL)-4, and fibroblast growth factor (FGF)-2. Compared to baseline, resveratrol increased plasma MMP10 and decreased IL-12P40, IL12P70, and RANTES. In this subset analysis, resveratrol treatment attenuated declines in mini-mental status examination (MMSE) scores, change in ADL (ADCS-ADL) scores, and CSF A 42 levels during the 52-week trial, but did not alter tau levels.

Conclusions: Collectively, these data suggest that resveratrol decreases CSF MMP9, modulates neuro-inflammation, and induces adaptive immunity. SIRT1 activation may be a viable target for treatment or prevention of neurodegenerative disorders.
\end{abstract}

Trial registration: ClinicalTrials.gov NCT01504854

Keywords: Resveratrol, Matrix metalloproteinase-(MMP)-9, Alzheimer, Interleukin-4, Macrophage-derived chemokine (MDC)

\section{Background}

Increasing age is the primary risk factor for Alzheimer's disease $(\mathrm{AD})$, even in individuals with high genetic risk. The mild stressor caloric restriction (CR) - or consuming $\sim 2 / 3$ normal daily calories-postpones and prevents diseases of aging in animal models and perhaps also in man. In contrast, diabetes mellitus and caloric excess (obesity, particularly during midlife) accelerate the

\footnotetext{
*Correspondence: cem46@georgetown.edu

'Department of Neurology, Laboratory for Dementia and Parkinsonism, Translational Neurotherapeutics Program, National Parkinson's Foundation Center of Excellence, Georgetown University Medical Center, 4000 Reservoi Road, NW, Washington DC 20057, USA

Full list of author information is available at the end of the article
}

onset of $\mathrm{AD}$, suggesting a link between glucose/energy metabolism and amyloid precursor protein $/ \beta$-amyloid (A $\beta$ ) metabolism. While the mechanism of CR benefits remains unclear, activation of sirtuins, notably SIRT1, may be a critical molecular pathway. SIRT1 deacetylase activity is regulated by NAD+/NADH-coupling cellular energy balance to epigenetic transcriptional regulation. Resveratrol, a potent SIRT1 activator and pharmacologic mimic of CR, is a polyphenol found naturally in red grapes, peanuts, and many other plant species. Similar to CR, treatment of transgenic mouse models of $\mathrm{AD}$ with resveratrol decreases behavioral deficits and central nervous system (CNS) $\mathrm{A} \beta$ deposition with aging [1]. 
We hypothesized that molecular mechanisms of aging, specifically SIRT1, may be exploited as a target for development of $\mathrm{AD}$ therapeutics. Given the proven safety of resveratrol and promising preclinical data, we enrolled 119 subjects in a phase 2 randomized, double-blind, placebo-controlled trial of resveratrol in subjects with mild-moderate AD (with dosage stepped up to $2 \mathrm{~g}$ pure, synthetic resveratrol by mouth daily, for 12 months) [2]. High-dose oral resveratrol treatment is safe and welltolerated-the only significant adverse effect is weight loss. Low nanomolar native resveratrol is detectable in cerebrospinal fluid (CSF), suggesting CNS penetration and a high-affinity molecular target (or targets). Compared to placebo, resveratrol stabilizes the progressive decline in CSF $A \beta 40$ and plasma $A \beta 40$ levels as dementia advances. In individuals with biomarker-confirmed AD (CSF A $342<600 \mathrm{ng} / \mathrm{ml}$ ) at baseline, resveratrol also stabilizes CSF A $\beta 42$ levels [2]. Despite the phase 2 trial being underpowered to detect clinical benefits, resveratrol attenuated decline in the Alzheimer's Disease Cooperative StudyActivity of Daily Living (ADCS-ADL) score during the 12month study. Aging is also a major risk factor for cancer, and fewer cancers were found in the resveratrol-treated group (one versus seven cancers in six participants in the placebo group). Collectively, these data support the notion that targeting molecular mechanisms of aging may point to therapeutic strategies that postpone or prevent diseases of aging-in parallel. With proven safety and suggestions of efficacy in the phase 2 trial, the putative benefits of resveratrol and other sirtuin activator compounds (STACs) should be further examined in clinical studies.

Paradoxically, resveratrol treatment increased brain volume loss in AD subjects, compared to the placebotreated group. Since CSF tau and phospho-tau levels are unaffected (suggesting no treatment effect on neuronal loss), we hypothesize that resveratrol has potent antiinflammatory effects in AD brain-with decreased CNS edema as the etiology of greater brain volume loss. Similar effects are found with anti-amyloid immunotherapies for $\mathrm{AD}$ [3] and effective drugs for multiple sclerosis (MS) are also known to be associated with "pseudoatrophy" [4]. To test the putative anti-inflammatory effects of resveratrol in AD brain, we measured pro- and anti-inflammatory cytokines and chemokines, and metalloproteinases, in banked samples of CSF and plasma from a subset of individuals with biomarker-confirmed AD (CSF A $342<600 \mathrm{ng} / \mathrm{ml}$ ) in the phase 2 trial. Consistent with our hypothesis, we found significant anti-inflammatory effects of resveratrol in the CSF of treated AD subjects. Our data also suggest that resveratrol treatment preserved the integrity of the bloodbrain barrier (BBB) in $\mathrm{AD}$. Collectively, these exploratory findings lend support to the notion that targeting molecular pathways of aging may lead to novel therapies to postpone or prevent diseases of aging, including AD.

\section{Methods}

\section{Patient demographics}

With the Alzheimer's Disease Cooperative Study, we recently completed a randomized, placebo-controlled, double-blind, multi-site, phase 2 trial of resveratrol in individuals with mild to moderate dementia due to AD [2]. The study drug was pure, synthetic resveratrol powder (encapsulated) versus matching placebo. Concomitant use of FDA-approved medications for AD (e.g., cholinesterase inhibitors) was allowed. The two randomized groups were similar at baseline with the exception that duration of diagnosis was longer in the placebo group. Participants (total $N=119$ ) were randomized to placebo or resveratrol $500 \mathrm{mg}$ orally once daily (with a dose escalation by 500-mg increments every 13 weeks, ending with $1000 \mathrm{mg}$ twice daily). The total treatment duration was 52 weeks. Dropout was less than anticipated, with $N=56$ completing week 52 in the resveratrol arm and $N=48$ completing week 52 in the placebo arm. Outcomes included safety and tolerability as well as effects on AD biomarkers (plasma $A \beta 40$ and $\mathrm{A} \beta 42$, CSF $\mathrm{A} \beta 40, \mathrm{~A} \beta 42$, tau, and phospho-tau181) and volumetric MRI (primary outcomes). Clinical outcomes (secondary) were also examined. Detailed pharmacokinetics were obtained in a subset $(n=15)$ at baseline and at weeks $13,26,39$, and 52 . As expected, oral resveratrol was rapidly metabolized with limited bioavailability. However, resveratrol and its major metabolites were measurable in plasma and CSF-demonstrating penetration of the blood-brain barrier. The only significant adverse event was weight loss. Compared to a decline found in the placebo group, plasma A $\beta 40$ and CSF A $\beta 40$ levels were stabilized by resveratrol. In the subset of individuals with biomarker-confirmed $\mathrm{AD}$ (baseline A $\beta 42<600 \mathrm{ng} / \mathrm{ml}$ ), resveratrol treatment also stabilized CSF A $\beta 42$. Brain volume loss was increased by resveratrol treatment (3 versus 1\%), suggesting a potent anti-inflammatory effect. The activities of daily living scale demonstrated less decline with resveratrol treatment, but the phase 2 study was inadequately powered to determine clinical outcomes. High-dose oral resveratrol is safe and well-tolerated in older individuals with AD. Further studies are needed to interpret the clinical and biomarker changes associated with resveratrol treatment.

\section{Human Neurodegenerative Disease Magnetic Bead Panels} We used a multiplex Xmap technology that uses magnetic microspheres internally coded with two fluorescent dyes to measure markers of neurodegeneration (Millipore, Cat\#: HNABTMAG-68K). All samples including placebo and resveratrol at baseline and 52 weeks were analyzed in parallel using the same reagents. Through precise combinations of these two dyes, multiple proteins are measured within the sample. Each of these spheres is coated with a 
specific capture antibody. The capture antibody binds to the detection antibody and a reporter molecule, completing the reaction on the surface of the bead. CSF or plasma $(25 \mu \mathrm{l})$ was incubated overnight at $4{ }^{\circ} \mathrm{C}$ with $25 \mu \mathrm{l}$ of a mixed bead solution, containing human total tau, ptau181, $A \beta 42$, and $A \beta 40$ (CSF $A \beta 40$ is diluted 1:10). After washing, samples were incubated with $25 \mu$ ldetection antibody solution for $1.5 \mathrm{~h}$ at room temperature. Streptavidin-phycoerythrin $(25 \mu \mathrm{l})$ was added to each well containing the $25 \mu \mathrm{l}$ of detection antibody solution. Samples were then washed and suspended in $100 \mu \mathrm{l}$ of sheath fluid. Samples were then run on MAGPIX with Xponent software. The median fluorescent intensity (MFI) data was analyzed using a 5-parameter logistic or spline curvefitting method for calculating analyte concentrations in samples. We also performed multiplex ELISA (Millipore, CAT\#: HCYTOMAG-60K) to profile a panel of plasma and CSF markers that are indicative of inflammation, including human EGF, FGF-2, Eotaxin, TGF- $\alpha$, G-CSF, Flt-3L, GM-CSF, Fractalkine, IFN $\alpha 2$, IFN $\gamma$, GRO, IL-10, MCP-3, IL-12P40, MDC, IL-12P70, PDGF-AA, IL-13, PDGF-AB/BB, IL-15, sCD40L, IL-17A, IL-1RA, IL-1 $\alpha$, IL9. IL-1 $\beta$, IL-2, IL-3, IL-4, IL-5, IL-6, IL-7, IL-8, IP-10, MCP-1, MIP- $1 \alpha$, MIP-1 $\beta$, RANTES, TNF $\alpha$, TNF $\beta$, and VEGF.

\section{Matrix metalloprotease ELISA}

Xmap technology uses magnetic microspheres that are internally coded with two fluorescent dyes. Through precise combinations of these two dyes, multiple proteins are simultaneously measured within a sample. Each of these spheres is coated with a specific capture antibody. The capture antibody binds to the detection antibody and a reporter molecule, completing the reaction on the surface of the bead. All samples including placebo and resveratrol at baseline and 52 weeks were analyzed in parallel using the same reagents. A total of $25 \mu \mathrm{l}$ human CSF or plasma was incubated overnight at $4{ }^{\circ} \mathrm{C}$ with $25 \mu \mathrm{l}$ of a mixed bead solution, containing human matrix metalloproteinase (MMP)-3, MMP-12, and MMP-13 (Millipore Cat\# HMMP1MAG-55K) or human MMP-1, MMP-2, MMP-7, MMP-9, and MMP-10 (Millipore Cat\# HMMP2MAG-55K). Following extensive washing of the plate, samples were incubated with $25 \mu$ of detection antibody solution for $1.5 \mathrm{~h}$ at room temperature and $25 \mu \mathrm{l}$ of streptavidin-phycoerythrin was added to each well. Samples were then washed and suspended in $100 \mu \mathrm{l}$ of sheath fluid. Samples were then run on MAGPIX with Xponent software. The median fluorescent intensity (MFI) data was analyzed using a five-parameter logistic or spline curve-fitting method for calculating analyte concentrations in samples according to manufacturer's protocols.

\section{Statistical analysis}

The inflammatory outcomes measured here are all exploratory, post hoc analyses. Data are summarized as raw values, range as appropriate, and mean \pm SD for $N=19$ in the placebo group and $N=19$ in the resveratrol group, unless otherwise indicated. All graphs and statistical analyses were performed in Graph Pad Prism Software version 5.01 (Graph Pad Prism Software, Inc. CA. USA). For baseline comparison between the two treatment arms, unpaired $t$ tests assuming both equal and unequal variances and Wilcoxon rank sum tests were performed to compare biomarkers and clinical variables. For categorical variables, Pearson's $\chi^{2}$ tests were used for comparison. Paired $t$ tests were performed within groups at baseline versus 52 weeks of treatment, and unpaired $t$ tests were performed for comparison of placebo and resveratrol treatment. We also fitted simple linear regression to see the associations between cognitive score (MMSE) and each biomarker among all of these individuals. The Benjamini and Hochberg (BH) multiple test correction is applied to control the false discovery rate at $0.05 . p$ values ("indicates statistical significance after $\mathrm{BH}$ adjustment) are summarized in Tables 1 and 2.

\section{Standard protocol approvals, registrations, and patient consents}

This study was conducted in accordance with Good Clinical Practice guidelines. Informed consent was obtained from participants and study partners. The study was conducted under local institutional review board supervision, under Food and Drug Administration IND 104205, and registered at ClinicalTrials.gov (NCT01504854).

\section{Results \\ CSF biomarkers}

At baseline, the levels of CSF biomarkers between the placebo group and resveratrol group were not significantly different (Table 1). The level of CSF MMP9 was significantly reduced in the placebo group between baseline and 52 weeks (Fig. 1a), and MMP9 was further reduced (48\%) at 52 weeks in the resveratrol group. No change in MMP9 was detected in the plasma (Table 2). Additionally, the level of interleukin (IL)-4 did not change in the placebo group, but CSF IL-4 was increased (Fig. 1b) in the resveratrol group. The CSF levels of macrophage-derived chemokine (MDC) (Fig. 1c) and fibroblast growth factor (FGF)-2 (Fig. 1d) were also increased after 52 weeks of resveratrol treatment compared to baseline, with no changes in these molecules in plasma (Table 1). There was no change in total CSF tau or hyper-phosphorylated ( $\mathrm{p}$-tau)181 levels in the resveratrol group and other inflammatory markers (Table 1) did not change. The level of CSF A $\beta 42$ was significantly reduced in the placebo (Fig. 1e) and resveratrol group at 52 weeks compared to baseline, consistent with our 
Table 1 Summary of statistical tests of null changes between baseline and 52 weeks and tests of null differences at baseline using all detected molecules in CSF of patients treated with placebo $(N=19)$ or resveratrol $(N=19)$

\begin{tabular}{|c|c|c|c|c|c|c|c|c|}
\hline \multirow[t]{3}{*}{ Analytes } & \multicolumn{5}{|c|}{ Between baseline and 52 weeks } & \multicolumn{3}{|l|}{ At baseline } \\
\hline & \multicolumn{2}{|c|}{ Paired $t$ test } & \multirow[t]{2}{*}{$\begin{array}{l}\text { Unpaired } \\
t \text { test }\end{array}$} & \multicolumn{2}{|c|}{$\begin{array}{l}\text { Wilcoxon signed } \\
\text { rank test }\end{array}$} & \multicolumn{3}{|l|}{ Active vs placebo } \\
\hline & Placebo & Active & & Placebo & Active & Unpaired $t$ test (unequal) & Unpaired $t$ test (equal) & Wilcoxon signed rank test \\
\hline$\overline{A \beta 40}$ & $0.0031^{* *}$ & $0.0014^{* *}$ & 0.0784 & $0.0066^{* *}$ & $0.0029 * *$ & 0.3579 & 0.3587 & 0.5284 \\
\hline$A \beta 42$ & $0.0112^{*}$ & $0.0027^{* *}$ & 0.0784 & $0.0105^{*}$ & $0.0034^{* *}$ & 0.3764 & 0.3739 & 0.4986 \\
\hline pTau 181 & 0.0665 & 0.6498 & 0.4409 & 0.0649 & 0.2891 & 0.7043 & 0.7044 & 0.9319 \\
\hline tTau & 0.91 & 0.3673 & 0.4614 & 0.9622 & 0.2163 & 0.5769 & 0.5638 & 0.9373 \\
\hline MMP-9 & $0.0474^{*}$ & $0.0027^{* *}$ & $0.0241^{*}$ & 0.0771 & $0.0034^{* *}$ & 0.8751 & 0.8737 & 1.0000 \\
\hline MMP-3 & 0.6098 & 0.5437 & 0.942 & 0.5016 & 0.6848 & 0.6911 & 0.6882 & 0.9311 \\
\hline MMP-2 & 0.5781 & 0.2322 & 0.9759 & 0.7049 & 0.3388 & 0.8828 & 0.8828 & 0.8509 \\
\hline MMP-10 & 0.4965 & 0.3175 & 0.9999 & 0.9341 & 0.5935 & 0.9415 & 0.9410 & 0.6807 \\
\hline FGF-2 & 0.3676 & $0.0248^{*}$ & 0.269 & 0.2128 & $0.0273^{*}$ & 0.6751 & 0.6774 & 0.5508 \\
\hline TGFa & 0.8693 & 0.0829 & 0.4257 & 0.61 & 0.2324 & 0.5571 & 0.5519 & 0.9843 \\
\hline G-CSF & 0.0532 & 0.1196 & 0.6722 & 0.0942 & 0.1563 & 0.2042 & 0.2422 & 0.4195 \\
\hline MCP-3 & 0.4751 & 0.0802 & 0.2922 & 0.5625 & 0.1094 & 0.2366 & 0.2305 & 0.2948 \\
\hline MDC & 0.8121 & $0.001 * * *$ & 0.0505 & 0.8938 & $0.0009^{* * *}$ & 0.0458 & 0.0566 & 0.0678 \\
\hline PDGF-AA & 0.9866 & 0.1106 & 0.0728 & 0.804 & 0.0787 & 0.9883 & 0.9886 & 0.8957 \\
\hline IL-4 & 0.9647 & $0.0085^{* *}$ & 0.1828 & 0.6875 & $0.0167^{*}$ & 0.0860 & 0.0992 & 0.0748 \\
\hline PDGF-AB/BB & 0.1752 & 0.4337 & 0.3356 & $0.0463^{*}$ & 0.057 & 0.6349 & 0.6261 & 0.7881 \\
\hline IL-8 & 0.262 & 0.1177 & 0.1594 & 0.57 & $0.0465^{*}$ & 0.9648 & 0.9648 & 0.6450 \\
\hline Flt-3L & 0.9602 & 0.1132 & 0.5639 & 0.8498 & 0.155 & 0.8880 & 0.8880 & 0.8109 \\
\hline Fractalkine & 0.791 & 0.4409 & 0.5973 & 0.6701 & 0.9382 & 0.4541 & 0.4543 & 0.8641 \\
\hline IFNa2 & 0.8832 & 0.4182 & 0.2943 & 0.8361 & 0.7547 & 0.8727 & 0.8729 & 0.6194 \\
\hline IL-15 & 0.9469 & 0.3587 & 0.3867 & 0.816 & 0.4263 & 0.7049 & 0.7029 & 0.6813 \\
\hline IL-7 & 0.8148 & 0.4458 & 0.3147 & 0.8384 & 1 & 0.4650 & 0.4553 & 0.4305 \\
\hline IP-10 & 0.2769 & 0.4091 & 0.405 & 0.6026 & 0.7368 & 0.3900 & 0.3918 & 0.8509 \\
\hline MCP-1 & 0.8089 & 0.5809 & 0.7419 & 0.421 & 0.8971 & 0.8650 & 0.8650 & 0.9864 \\
\hline MIP-1b & 0.3973 & 0.2909 & 0.6174 & 0.3621 & 0.2188 & 0.7722 & 0.7632 & 0.8401 \\
\hline
\end{tabular}

Indicated in bold typeface represents significant associations (at level 0.05) after the Benjamini-Hochberg correction

${ }^{*} p<0.05$

${ }^{* *} p<0.1$

*** $p<0.001$

previous data [2]. However, the decline of CSF A $\beta 42$ in the placebo group was greater than the decline in the resveratrol group $(p=0.0618)$. Furthermore, CSF A $\beta 40$ was significantly reduced in the resveratrol group at 52 weeks compared to baseline (Fig. 1f). Multiple test corrections to control for a false discovery rate $<0.05$ were performed and the significant associations in CSF markers were unchanged after this analysis.

\section{Plasma biomarkers}

At baseline plasma level of each biomarker between the placebo group and resveratrol group were not significantly different (Table 2). The plasma level of MMP10 was increased at 52 weeks of resveratrol treatment compared to baseline and placebo (Fig. 2a), and MMP10 did not change in CSF (Table 1). MMP3 and MMP2 did not change in the CSF (Table 1) or plasma and MMP1,
MMP12, and MMP13 did not change in plasma (Table 2). Plasma IL-1R4 (Fig. 2b) and IL-12P40 (Fig. 2c) were increased at 52 weeks compared to baseline in the placebo group, but this increase was slightly reduced in the resveratrol group. The plasma levels of IL-12P70 (Fig. 2d) did not change with placebo but was reduced at 52 weeks compared to baseline in the resveratrol group before multiple test adjustment. Plasma tumor necrosis factor (TNF)- $\alpha$ (Fig. 2e) was increased at 52 weeks compared to baseline with placebo and did not change in the resveratrol group. Plasma levels of RANTES/CCL5 (Fig. 2f) did not change with placebo but was reduced at 52 weeks compared to baseline in the resveratrol group. The plasma level of IL-8 (Table 2) was reduced at 52 weeks in the resveratrol group compared to placebo. No changes were observed in other markers (Tables 1 and 2) between groups. However, statistical associations in plasma markers 
Table 2 Summary of statistical tests of null changes between baseline and 52 weeks and tests of null differences at baseline using all detected molecules in plasma of patients treated with placebo $(N=19)$ or resveratrol $(N=19)$

\begin{tabular}{|c|c|c|c|c|c|c|c|c|}
\hline \multirow[t]{3}{*}{ Analytes } & \multicolumn{5}{|c|}{ Between baseline and 52 weeks } & \multicolumn{3}{|l|}{ At baseline } \\
\hline & \multicolumn{2}{|c|}{ Paired $t$ test } & \multirow[t]{2}{*}{ Unpaired $t$ test } & \multicolumn{2}{|c|}{$\begin{array}{l}\text { Wilcoxon signed rank } \\
\text { test }\end{array}$} & \multicolumn{3}{|l|}{ Baseline: active vs placebo } \\
\hline & Placebo & Active & & Placebo & Active & Unpaired $t$ test (unequal) & Unpaired $t$ test (equal) & Wilcoxon signed rank test \\
\hline MMP-9 & 0.1477 & 0.8332 & 0.7577 & 0.2412 & 0.6319 & 0.8751 & 0.8737 & 1.0000 \\
\hline MMP-3 & 0.3193 & $0.0062^{* *}$ & 0.065 & 0.3575 & $0.0102^{*}$ & 0.6911 & 0.6882 & 0.9311 \\
\hline MMP-2 & 0.5783 & 0.1647 & 0.1671 & 0.7148 & 0.177 & 0.8828 & 0.8828 & 0.8509 \\
\hline MMP-10 & 0.4557 & $0.0243^{*}$ & $0.0106^{*}$ & 0.5417 & 0.0353 & 0.9415 & 0.9410 & 0.6807 \\
\hline MMP-12 & 0.457 & 0.9138 & 0.4917 & 0.4631 & 0.9306 & 0.3667 & 0.3787 & 0.3310 \\
\hline MMP-13 & 0.1897 & 0.3379 & $0.0489^{*}$ & 0.3575 & 0.421 & 0.0598 & 0.0722 & 0.0938 \\
\hline MMP-1 & 0.7483 & 0.9518 & 0.7139 & 0.4263 & 0.8129 & 0.1371 & 0.1264 & 0.1059 \\
\hline FGF-2 & 0.6374 & 0.9743 & 0.6426 & 0.7002 & 0.6701 & 0.6751 & 0.6774 & 0.5508 \\
\hline G-CSF & 0.3334 & 0.4526 & 0.2682 & 0.4131 & 0.5703 & 0.2042 & 0.2422 & 0.4195 \\
\hline MDC & 0.7177 & 0.8208 & 0.1778 & 0.7148 & 0.7605 & 0.0458 & 0.0566 & 0.0678 \\
\hline PDGF-AA & 0.8196 & 0.5719 & 0.3956 & 0.583 & 0.5421 & 0.9883 & 0.9886 & 0.8957 \\
\hline PDGF-AB/BB & 0.2176 & 0.889 & 0.998 & 0.3258 & 0.9246 & 0.6349 & 0.6261 & 0.7881 \\
\hline IL-8 & 0.2096 & 0.7207 & $0.0322^{*}$ & 0.5416 & 0.9794 & 0.9648 & 0.9648 & 0.6450 \\
\hline EGF & 0.5238 & 0.5667 & 0.7799 & 0.4263 & 0.8564 & 0.8870 & 0.8815 & 0.7234 \\
\hline Eotaxin & 0.5987 & 0.0518 & 0.0697 & 0.5016 & 0.0611 & 0.7968 & 0.8017 & 0.8212 \\
\hline GM-CSF & 0.6863 & 0.4321 & 0.3562 & 0.8501 & 0.4648 & 0.5001 & 0.4856 & 0.6638 \\
\hline Fractalkine & 0.8863 & 0.8397 & 0.8579 & 0.9032 & 0.9245 & 0.4541 & 0.4543 & 0.8641 \\
\hline IFNa2 & 0.8981 & 0.8459 & 0.9661 & 1 & 0.9622 & 0.8727 & 0.8729 & 0.6194 \\
\hline IFNy & 0.8316 & 0.438 & 0.8992 & 0.7148 & 0.463 & 0.8357 & 0.8406 & 0.8745 \\
\hline GRO & 0.3481 & 0.6638 & 0.8992 & 0.2958 & 0.9622 & 0.9496 & 0.9478 & 0.5825 \\
\hline IL-12P40 & 0.6103 & $0.0485^{*}$ & 0.6473 & 0.9102 & 0.0781 & 0.2033 & 0.1897 & 0.3816 \\
\hline IL-12P70 & 0.5558 & $0.0404^{*}$ & 0.8159 & 0.7344 & $0.0424^{*}$ & 0.6327 & 0.6141 & 0.7730 \\
\hline sCD40L & 0.3657 & 0.793 & 0.9062 & 0.3258 & 0.9653 & 0.6623 & 0.6483 & 0.8802 \\
\hline IL-17A & 0.3792 & 0.7593 & 0.1786 & 0.4752 & 0.8158 & 0.3152 & 0.2696 & 0.6923 \\
\hline IL-1RA & $0.0462^{*}$ & 0.4993 & 0.6706 & $0.0137^{*}$ & 0.7615 & 0.0880 & 0.0802 & 0.0317 \\
\hline IL-2 & 0.8926 & $0.0311^{*}$ & 0.9868 & 1 & 0.0547 & 0.2103 & 0.2015 & 0.2712 \\
\hline IP-10 & 0.1619 & 0.0673 & 0.0975 & 0.1353 & 0.0742 & 0.3900 & 0.3918 & 0.8509 \\
\hline MCP-1 & 0.5574 & 0.0637 & 0.2533 & 0.7148 & 0.0523 & 0.8650 & 0.8650 & 0.9864 \\
\hline MIP-1a & 0.3696 & 0.2241 & 0.588 & 0.3594 & 0.1289 & 0.0175 & 0.0247 & 0.0440 \\
\hline MIP-1b & 0.3694 & 0.6581 & 0.5786 & 0.3652 & 0.8469 & 0.7722 & 0.7632 & 0.8401 \\
\hline RANTES & 0.8545 & $0.0335^{*}$ & 0.2919 & 0.9515 & $0.0366^{*}$ & 0.9889 & 0.9889 & 0.9699 \\
\hline TNFa & $0.0221^{*}$ & 0.2091 & 0.7741 & $0.0161^{*}$ & 0.463 & 0.4081 & 0.4298 & 0.5861 \\
\hline
\end{tabular}

Indicated in bold typeface represents significant associations (at level 0.05) after the Benjamini-Hochberg correction

${ }^{*} p<0.05$

${ }^{* *} p<0.1$

did not hold after multiple test correction, suggesting that samples from a larger number of subjects may be required to discover putative significant effects.

\section{Cognitive outcomes}

A reduction in mini-mental score examination (MMSE) scores was observed at 52 weeks compared to baseline in the placebo group (Fig. 3a, $p<0.01$ ), but no significant change was detected in MMSE between baseline and
52 weeks with resveratrol treatment. ADCS-ADL scores showed a decline at 52 weeks compared to control (Fig. 3b) in both placebo $(p<0.001)$ and resveratrol $(p<0.001)$ groups; however, the decline in placebo was twofold greater than resveratrol at week 52 (Fig. 3c), suggesting that resveratrol may slow progressive cognitive and functional decline in mild to moderate AD subjects. There is no statistically significant association between the change in MMSE and change in each of 

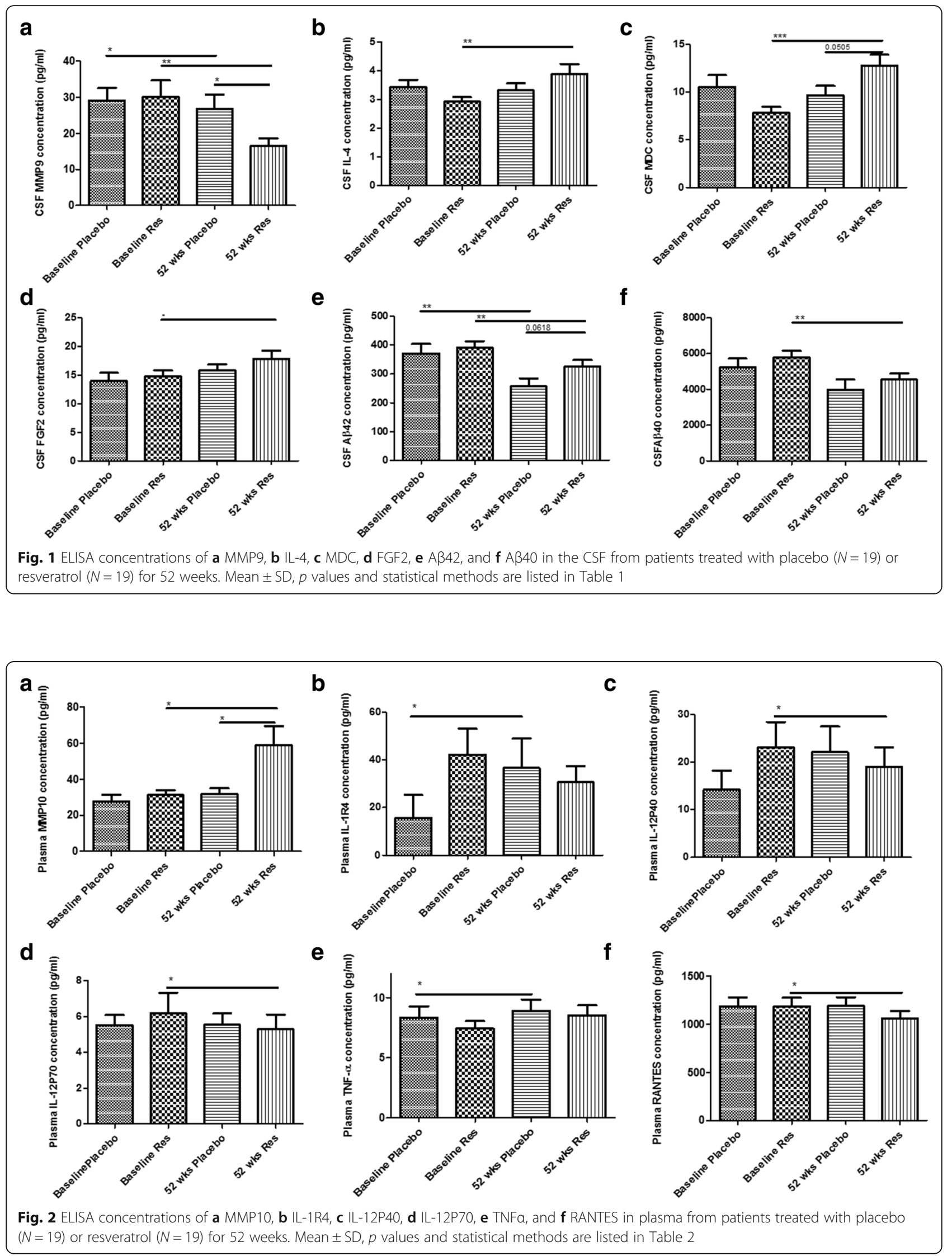


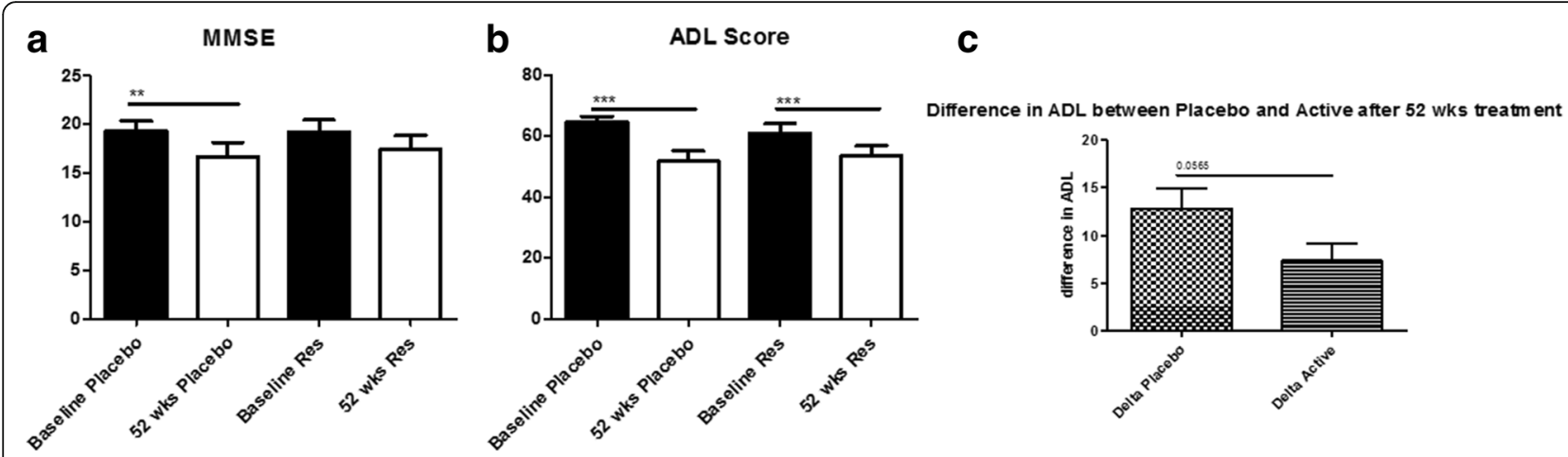

Fig. 3 Histograms represent a MMSE scores and $\mathbf{b}$ ADCS-ADL and $\mathbf{c}$ changes in ADL in placebo versus resveratrol groups in patients treated with placebo $(N=19)$ or resveratrol $(N=19)$ for 52 weeks. Mean $\pm S D,{ }^{* *} p<0.01,{ }^{* * *} p<0.001$

CSF or plasma biomarker between baseline and 52 weeks (Table 3).

\section{Discussion}

One of the most striking results of this study is the significant decrease in the level of CSF MMP9 after resveratrol treatment. MMP9 has recently emerged as a major player in several brain pathologies, including neurodegeneration and neuro-inflammation [5]. MMP9 regulates $\mathrm{BBB}$ permeability via release of cytokines and free radicals as well as cleavage of vascular basal lamina and/or tight junctions in the neurovascular unit in both MS and AD [6-8]. The decrease in CSF MMP9 levels suggests that resveratrol treatment may reduce $\mathrm{CNS}$ permeability and limit the infiltration of leukocytes and other inflammatory agents into the brain. MMP9-mediated breakdown of the basal lamina and destruction of gap junctions in the neurovascular unit result in increased CNS permeability and inflammation in autoimmune encephalitis, hypoxic brain injury, and other diseases $[9,10]$. MMP9 knockout reduces neuro-inflammation in experimental autoimmune encephalomyelitis (EAE) [11], while CSF MMP9 is elevated in patients with bacterial meningitis and $\mathrm{BBB}$ damage [12]. Moreover, inhibition of MMP9 alleviates the neurological damage associated with human immunodeficiency virus (HIV) infection [13], suggesting that MMP9 activation is a response to HIV infection. These data are also supported by enhanced expression and activity of MMP9 in serum, CSF, and demyelinating lesions in MS [14], and abundant evidence of increased MMP9 expression and activity in ischemic stroke $[15,16]$. Animal studies have also revealed significant increases in MMP9 levels after traumatic brain injury [17], but damage to the BBB and behavioral deficits are significantly attenuated in MMP9 knockout animals $[18,19]$.

MMP9 is highly regulated both spatially and temporally with many target substrates including growth factors, cell surface receptors, and cell adhesion molecules. Low levels of MMP9 messenger RNA (mRNA) and protein expression are detected predominantly in neurons in the hippocampus, cerebellum and cerebral cortex of normal brain [20], but injury significantly increases the mRNA and protein levels and activity of MMP9 [5, 21, 22], which may be derived from brain cells or leukocyte invasion of the brain due to BBB compromise. Intercellular adhesion molecule-5 (ICAM-5), which mediates the regulation of dendritic spine elongation and maturation may be cleaved by MMP9 upon activation of N-methyl-D-aspartate (NMDA) receptors $[23,24]$, suggesting a role for MMP9 in synaptic function. Furthermore, MMP9 deletion increases the number of CA1 pyramidal neurons and decreases the length and complexity of dendritic spines [25]. Immune system dysfunction may develop with aging in parallel with upregulation of brain MMP9 [26-28]. However, a recent study showed that CSF MMP9 was significantly lower in $A D$ subjects with decreased $A \beta 42$ and $A \beta 40$ and increased total tau and p-tau levels compared to healthy controls [29]. In the current study, the levels of CSF tau and p-tau were not altered by treatment but the levels of CSF A $\beta 42$ and $A \beta 40$ were altered in parallel with a reduction of MMP9. However, there was no difference in the level of CSF MMP9 between placebo and resveratrol-treated groups at baseline, and it is uncertain whether MMP9 in our study population with $\mathrm{AD}$ is different from healthy controls. MMP9 activation is likely driven by other MMPs [30], so we examined the level of MMPs in plasma and CSF. Leukocyte penetration into brain parenchyma in EAE models involves $\beta$-dystoglycan cleavage that is only abolished in double MMP2 and MMP9 knockout mice [31], suggesting the effects of other MMPs on MMP9 function. MMP10 and MMP3 were slightly increased in the plasma but not CSF of AD patients. MMP9 has overlapping substrates with other MMPs that share similar structures [5], so caution must be used in the interpretation of specific MMP9 targets. MMP9 also plays a role in post-natal brain development during a critical period of synaptic formation and maturation and axonal myelination [32]. 
Table 3 Summary of statistical tests of associations between changes in MMSE for 52 weeks and changes in each biomarker for 52 among patients treated with placebo $(N=19)$ or resveratrol $(N=19)$

\begin{tabular}{|c|c|c|c|}
\hline \multicolumn{4}{|c|}{ Association between changes in MMSE and changes in biomarkers } \\
\hline CSF & $p$ value & Plasma & $p$ value \\
\hline$A \beta 40$ & 0.8367 & MMP-9 & 0.1061 \\
\hline$A \beta 42$ & 0.2180 & MMP-3 & 0.1358 \\
\hline pTau 181 & 0.4521 & MMP-2 & 0.4234 \\
\hline tTau & 0.6607 & MMP-10 & 0.6379 \\
\hline MMP-9 & 0.1061 & MMP-12 & 0.9992 \\
\hline MMP-3 & 0.1358 & MMP-13 & 0.5432 \\
\hline MMP-2 & 0.4234 & MMP-1 & 0.9490 \\
\hline MMP-10 & 0.6379 & FGF-2 & 0.5382 \\
\hline FGF-2 & 0.5382 & G-CSF & 0.3103 \\
\hline TGFa & 0.1270 & MDC & 0.7157 \\
\hline G-CSF & 0.3103 & PDGF-AA & 0.2266 \\
\hline MCP-3 & 0.8066 & PDGF-AB/BB & 0.8598 \\
\hline MDC & 0.7157 & IL-8 & 0.0705 \\
\hline PDGF-AA & 0.2266 & EGF & 0.3491 \\
\hline IL-4 & 0.7163 & Eotaxin & 0.2782 \\
\hline PDGF-AB/BB & 0.8598 & GM-CSF & 0.9380 \\
\hline IL-8 & 0.0705 & Fractalkine & 0.4894 \\
\hline Flt-3L & 0.2061 & IFNa2 & 0.9855 \\
\hline Fractalkine & 0.4894 & IFNy & 0.3895 \\
\hline IFNa2 & 0.9855 & GRO & 0.2955 \\
\hline IL-15 & 0.8288 & IL-12P40 & 0.7747 \\
\hline IL-7 & 0.8204 & IL-12P70 & 0.8371 \\
\hline IP-10 & 0.2102 & sCD40L & 0.3075 \\
\hline MCP-1 & 0.2516 & IL-17A & 0.8985 \\
\hline \multirow[t]{8}{*}{ MIP-1b } & 0.5144 & IL-1RA & 0.5109 \\
\hline & & $\| \mathrm{L}-2$ & 0.6367 \\
\hline & & IP-10 & 0.2102 \\
\hline & & MCP-1 & 0.2516 \\
\hline & & MIP-1a & 0.6162 \\
\hline & & MIP-1b & 0.5144 \\
\hline & & RANTES & 0.4541 \\
\hline & & TNFa & 0.2814 \\
\hline
\end{tabular}

Indicated in bold typeface represents significant associations (at level 0.05) after the Benjamini-Hochberg correction

In the adult brain MMP9 and MMP3 may be involved in neurogenesis and migratory response mechanisms [33]. MMP9 is upregulated in delayed and acute phases of post ischemic stroke models [34, 35].

MMP9 regulates the CNS immune response due to its ability to activate inflammatory markers and its involvement in $\mathrm{BBB}$ maintenance, leading to its regulation of entry of leukocytes into the brain parenchyma [5]. MDC/
CCL22 is a small cytokine that belongs to the CysteineCysteine (CC) family and is involved in transport of natural killer cells, chronically activated $\mathrm{T}$ lymphocytes (Th2) [36], monocytes, and dendritic cells into injury sites [37]. MDC is expressed in the CNS and is produced by CNS-infiltrating leukocytes and intra-parenchymal microglia in EAE models [38]. Activated microglia secrete MDC that induces chemotaxis of Th2, but not Th1, cells suggesting that MDC produced by microglia regulates neuroinflammation via recruitment of Th2 cells into the injury site [38]. Leukocyte infiltration into CNS white matter lesions, which contain $\mathrm{CD}^{+}$and $\mathrm{CD}^{+} \mathrm{T}$ cells and activated macrophages/microglia, is a hallmark of MS [39]. Taken together, these findings support the hypothesis that the increase in CNS MDC with resveratrol may facilitate the intracerebral homing of specific leukocytes involved in brain injury in $\mathrm{AD}$, providing a mechanism for responding to amyloid-associated inflammation [40]. MDC is involved in Th2-driven chronic inflammation [41], and this is consistent with the increase of CNS levels of IL-4, which mediates an adaptive immune response via Th2 cell induction [42, 43], leading to a long-term protective immune response. Our results are also consistent with the function MMPs that play an integral role in immune cell development, effector function, migration, and ligand-receptor interactions [44]. T helper cells (Th1 and Th2) secrete MMP9 [45], which plays a critical role in the migration of $\mathrm{T}$ cells from the blood stream to the brain and other tissues [46, 47]. Additionally, recent advances in neuro-inflammation implicate abnormal neurotrophic factor signaling, including fibroblast growth factors (FGFs) in HIV-associated neurocognitive decline (HAND) [48] and stroke [49]. The increase in CNS FGF levels after resveratrol treatment suggests an effect on growth factors, which may play a role in neuro-resilience in aging and $\mathrm{AD}$.

Neuro-inflammation may contribute to cognitive impairment and play a significant role in AD progression. Activation of specific microglia/macrophage may be neuroprotective. Although resveratrol treatment did not affect CSF tau, resveratrol significantly attenuated the declines in CSF A $\beta 42$ and A $\beta 40$ levels (compared to placebo) and attenuated cognitive and functional decline (MMSE and ADCS-ADL) between the placebo and treated groups. Resveratrol also reduced the plasma levels of pro-inflammatory makers including IL-1R4, IL-12P40, IL-12P70, TNF- $\alpha$, and RANTES, independent of CSF changes of the levels of these biomarkers.

Innate immune cells, including CNS resident microglia and peripheral bone marrow-derived macrophages can exhibit a dysfunctional or senescent profile characterized by impaired phagocytosis as AD progresses, indicating that modification of the microglia/macrophage activation state, instead of inhibiting their function, may hold 
therapeutic promise in AD [50-52]. Resveratrol may facilitate activation of microglia/macrophages therefore inducing a long-term adaptive immune response that may be clinically beneficial in AD subjects. Major impediments of current immunotherapy approaches to $\mathrm{AD}$ include limited evidence of significant clinical benefits, and the risk of excessive neuro-inflammation [53].

\section{Conclusions}

Resveratrol may maintain the integrity of the $\mathrm{BBB}$ via reduction of MMP9 and induce adaptive immune responses that may promote brain resilience to amyloid deposition. Resveratrol may slow cognitive decline in $A D$ via a coordinated peripheral and central immune response that may also arrest neuronal death. In conclusion, the exploratory findings of the current study encourage further validation of the hypothesis that resveratrol may seal off a leaky BBB and contribute to cognitive and functional improvement in a larger follow-up study with AD patients.

\section{Abbreviations}

AD: Alzheimer's disease; BBB: Blood-brain barrier; CR: Caloric restriction; CSF: Cerebrospinal fluid; IL: Interleukin; MDC: Macrophage-derived chemokine; MMP: Matrix metalloproteinase; Res: Resveratrol; SIRT: Surtuin; TNF-a: Tumor necrosis factor-a

\section{Acknowledgements}

We also thank Louise Monte and Shannon Campbell at the ADCS Biomarker Core for their efforts in the original trial and provision of CSF and plasma samples.

\section{Funding}

This work was supported by NIA U01 AG010483 (to PSA) for original trial, Georgetown University support (to CM) to conduct the biomarker studies and write the manuscript, and a philanthropic gift from Ms. Pat Harvey (to RST) to purchase ELISA kits for biomarkers. The original trial may be found at ClinicalTrials.gov NCT01504854

\section{Availability of data and materials}

All experimental data and unique biological materials used in this study are available upon request.

\section{Authors' contributions}

CM supervised the processing of bio fluid samples, wrote the manuscript and analyzed the data. $\mathrm{M} \mathrm{H}$ and $\mathrm{X} \mathrm{H}$ conducted the ELISA. JA performed biostatistics. RAR and PSA provided the samples. RST is principal investigator on the Res trials and edited the manuscript. All authors read and approved the final manuscript.

\section{Competing interests}

The authors declare that they have no competing interests.

\section{Consent for publication}

Not applicable.

\section{Ethics approval and consent to participate}

CSF and plasma samples were collected with informed consent as a part of the Res clinical trial from patients who were enrolled in the clinical trial under Food and Drug Administration IND 104205, and registered at ClinicalTrials.gov (NCT01504854). All CSF and plasma samples were handled with strict anonymity throughout the study. The study was conducted under local institutional review board supervision.

\section{Author details}

Department of Neurology, Laboratory for Dementia and Parkinsonism, Translational Neurotherapeutics Program, National Parkinson's Foundation Center of Excellence, Georgetown University Medical Center, 4000 Reservoir Road, NW, Washington DC 20057, USA. ${ }^{2}$ Department of Neurology, Memory Disorders Program, Translational Neurotherapeutics Program, Georgetown University, Washington DC, USA. ${ }^{3}$ Department of Biostatistics, Georgetown University Medical Center, 4000 Reservoir Road, NW, Washington DC 20057, USA. ${ }^{4}$ Alzheimer's Therapeutic Research Institute (ATRI), University of Southern California, San Diego, CA, USA. ${ }^{5}$ Alzheimer's Disease Cooperative Study (ADCS), Department of Neurosciences, University of California, La Jolla, San Diego, CA, USA.

Received: 21 October 2016 Accepted: 13 December 2016

Published online: 03 January 2017

\section{References}

1. Pasinetti GM, Wang J, Ho L, Zhao W, Dubner L. Roles of resveratrol and other grape-derived polyphenols in Alzheimer's disease prevention and treatment. Biochim Biophys Acta. 2015:1852:1202-8.

2. Turner RS, Thomas RG, Craft S, van Dyck CH, Mintzer J, Reynolds BA, Brewer JB, Rissman RA, Raman R, Aisen PS, Alzheimer's Disease Cooperative S. A randomized, double-blind, placebo-controlled trial of resveratrol for Alzheimer disease. Neurology. 2015:85:1383-91.

3. Fox NC, Black RS, Gilman S, Rossor MN, Griffith SG, Jenkins L, Koller M, Study AN. Effects of Abeta immunization (AN1792) on MRI measures of cerebral volume in Alzheimer disease. Neurology. 2005;64:1563-72.

4. De Stefano N, Airas L, Grigoriadis N, Mattle HP, O'Riordan J, Oreja-Guevara C, Sellebjerg F, Stankoff B, Walczak A, Wiendl H, Kieseier BC. Clinical relevance of brain volume measures in multiple sclerosis. CNS Drugs. 2014;28:147-56.

5. Vafadari B, Salamian A, Kaczmarek L. MMP-9 in Translation: From Molecule to Brain Physiology, Pathology and Therapy. J Neurochem. 2016;139 (Suppl 2):91-114.

6. Verslegers M, Lemmens K, Van Hove I, Moons L. Matrix metalloproteinase-2 and -9 as promising benefactors in development, plasticity and repair of the nervous system. Prog Neurobiol. 2013;105:60-78.

7. Reijerkerk A, Kooij G, van der Pol SM, Khazen S, Dijkstra CD, de Vries HE. Diapedesis of monocytes is associated with MMP-mediated occludin disappearance in brain endothelial cells. FASEB J. 2006;20:2550-2.

8. Candelario-Jalil E, Yang Y, Rosenberg GA. Diverse roles of matrix metalloproteinases and tissue inhibitors of metalloproteinases in neuroinflammation and cerebral ischemia. Neuroscience. 2009;158:983-94.

9. Svedin P, Hagberg H, Savman K, Zhu C, Mallard C. Matrix metalloproteinase9 gene knock-out protects the immature brain after cerebral hypoxiaischemia. J Neurosci. 2007;27:1511-8.

10. Rosenberg GA. Matrix metalloproteinases in neuroinflammation. Glia. 2002; 39:279-91.

11. Dubois B, Masure $S$, Hurtenbach U, Paemen L, Heremans $H$, van den Oord J, Sciot R, Meinhardt T, Hammerling G, Opdenakker G, Arnold B. Resistance of young gelatinase $\mathrm{B}$-deficient mice to experimental autoimmune encephalomyelitis and necrotizing tail lesions. J Clin Invest. 1999;104: 1507-15.

12. Leppert D, Leib SL, Grygar C, Miller KM, Schaad UB, Hollander GA. Matrix metalloproteinase (MMP)-8 and MMP-9 in cerebrospinal fluid during bacterial meningitis: association with blood-brain barrier damage and neurological sequelae. Clin Infect Dis. 2000;31:80-4.

13. Gramegna P, Latronico T, Brana MT, Di Bari G, Mengoni F, Belvisi V, Mascellino MT, Lichtner M, Vullo V, Mastroianni CM, Liuzzi GM. In vitro downregulation of matrix metalloproteinase- 9 in rat glial cells by CCR5 antagonist maraviroc: therapeutic implication for HIV brain infection. PLoS One. 2011;6:e28499.

14. Yong WW, Zabad RK, Agrawal S, Goncalves Dasilva A, Metz LM. Elevation of matrix metalloproteinases (MMPs) in multiple sclerosis and impact of immunomodulators. J Neurol Sci. 2007:259:79-84.

15. Yang $Y$, Rosenberg GA. Matrix metalloproteinases as therapeutic targets for stroke. Brain Res. 2015:1623:30-8.

16. Chaturvedi M, Kaczmarek L. Mmp-9 inhibition: a therapeutic strategy in ischemic stroke. Mol Neurobiol. 2014:49:563-73.

17. Hayashi T, Kaneko Y, Yu S, Bae E, Stahl CE, Kawase T, van Loveren H, Sanberg PR, Borlongan CV. Quantitative analyses of matrix metalloproteinase activity after traumatic brain injury in adult rats. Brain Res. 2009;1280:172-7. 
18. Wang X, Jung J, Asahi M, Chwang W, Russo L, Moskowitz MA, Dixon CE, Fini ME, Lo EH. Effects of matrix metalloproteinase-9 gene knock-out on morphological and motor outcomes after traumatic brain injury. J Neurosci. 2000;20:7037-42.

19. Asahi M, Asahi K, Jung JC, del Zoppo GJ, Fini ME, Lo EH. Role for matrix metalloproteinase 9 after focal cerebral ischemia: effects of gene knockout and enzyme inhibition with BB-94. J Cereb Blood Flow Metab. 2000;20:1681-9.

20. Dzwonek J, Rylski M, Kaczmarek L. Matrix metalloproteinases and their endogenous inhibitors in neuronal physiology of the adult brain. FEBS Lett. 2004:567:129-35.

21. Michaluk P, Kolodziej L, Mioduszewska B, Wilczynski GM, Dzwonek J, Jaworski J, Gorecki DC, Ottersen OP, Kaczmarek L. Beta-dystroglycan as a target for MMP-9, in response to enhanced neuronal activity. J Biol Chem. 2007;282:16036-41.

22. Dziembowska M, Milek J, Janusz A, Rejmak E, Romanowska E, Gorkiewicz T, Tiron A, Bramham CR, Kaczmarek L. Activity-dependent local translation of matrix metalloproteinase-9. J Neurosci. 2012;32:14538-47.

23. Conant K, Wang Y, Szklarczyk A, Dudak A, Mattson MP, Lim ST. Matrix metalloproteinase-dependent shedding of intercellular adhesion molecule-5 occurs with long-term potentiation. Neuroscience. 2010;166:508-21.

24. Tian L, Stefanidakis M, Ning L, Van Lint P, Nyman-Huttunen H, Libert C, Itohara S, Mishina M, Rauvala H, Gahmberg CG. Activation of NMDA receptors promotes dendritic spine development through MMP-mediated ICAM-5 cleavage. J Cell Biol. 2007;178:687-700.

25. Murase S, Lantz CL, Kim E, Gupta N, Higgins R, Stopfer M, Hoffman DA, Quinlan EM. Matrix metalloproteinase-9 regulates neuronal circuit development and excitability. Mol Neurobiol. 2016;53:3477-93.

26. Romero JR, Vasan RS, Beiser AS, Au R, Benjamin EJ, DeCarli C, Wolf PA, Seshadri S. Association of matrix metalloproteinases with MRI indices of brain ischemia and aging. Neurobiol Aging. 2010;31:2128-35.

27. Safciuc F, Constantin A, Manea A, Nicolae M, Popov D, Raicu M, Alexandru D, Constantinescu E. Advanced glycation end products, oxidative stress and metalloproteinases are altered in the cerebral microvasculature during aging. Curr Neurovasc Res. 2007;4:228-34.

28. Mroczko B, Groblewska M, Barcikowska M. The role of matrix metalloproteinases and tissue inhibitors of metalloproteinases in the pathophysiology of neurodegeneration: a literature study. J Alzheimers Dis. 2013;37:273-83.

29. Mroczko B, Groblewska M, Zboch M, Kulczynska A, Koper OM, Szmitkowski $\mathrm{M}$, Kornhuber J, Lewczuk P. Concentrations of matrix metalloproteinases and their tissue inhibitors in the cerebrospinal fluid of patients with Alzheimer's disease. J Alzheimers Dis. 2014:40:351-7.

30. Stawarski M, Stefaniuk M, Wlodarczyk J. Matrix metalloproteinase-9 involvement in the structural plasticity of dendritic spines. Front Neuroanat. 2014:8:68.

31. Agrawal S, Anderson P, Durbeej M, van Rooijen N, Ivars F, Opdenakker G, Sorokin LM. Dystroglycan is selectively cleaved at the parenchymal basement membrane at sites of leukocyte extravasation in experimental autoimmune encephalomyelitis. J Exp Med. 2006;203:1007-19.

32. Reinhard SM, Razak K, Ethell IM. A delicate balance: role of MMP-9 in brain development and pathophysiology of neurodevelopmental disorders. Front Cell Neurosci. 2015;9:280

33. Barkho BZ, Munoz AE, Li X, Li L, Cunningham LA, Zhao X. Endogenous matrix metalloproteinase (MMP)-3 and MMP-9 promote the differentiation and migration of adult neural progenitor cells in response to chemokines. Stem Cells. 2008;26:3139-49.

34. Lee SR, Kim HY, Rogowska J, Zhao BQ, Bhide P, Parent JM, Lo EH. Involvement of matrix metalloproteinase in neuroblast cell migration from the subventricular zone after stroke. J Neurosci. 2006;26:3491-5.

35. Lu L, Tonchev AB, Kaplamadzhiev DB, Boneva NB, Mori Y, Sahara S, Ma D, Nakaya MA, Kikuchi M, Yamashima T. Expression of matrix metalloproteinases in the neurogenic niche of the adult monkey hippocampus after ischemia. Hippocampus. 2008;18:1074-84.

36. Imai T, Nagira M, Takagi S, Kakizaki M, Nishimura M, Wang J, Gray PW, Matsushima K, Yoshie O. Selective recruitment of CCR4-bearing Th2 cells toward antigen-presenting cells by the CC chemokines thymus and activation-regulated chemokine and macrophage-derived chemokine. Int Immunol. 1999;11:81-8.

37. Blobel CP. Metalloprotease-disintegrins: links to cell adhesion and cleavage of TNF alpha and Notch. Cell. 1997;90:589-92.

38. Columba-Cabezas S, Serafini B, Ambrosini E, Sanchez M, Penna G, Adorini L, Aloisi F. Induction of macrophage-derived chemokine/CCL22 expression in experimental autoimmune encephalomyelitis and cultured microglia: implications for disease regulation. J Neuroimmunol. 2002;130:10-21.

39. Wingerchuk DM, Lucchinetti CF, Noseworthy JH. Multiple sclerosis: current pathophysiological concepts. Lab Invest. 2001;81:263-81.

40. Glabinski AR, Ransohoff RM. Chemokines and chemokine receptors in CNS pathology. J Neurovirol. 1999;5:3-12.

41. Jugde F, Alizadeh M, Boissier C, Chantry D, Siproudhis L, Corbinais S, Quelvennec E, Dyard F, Campion JP, Gosselin M, et al. Quantitation of chemokines (MDC, TARC) expression in mucosa from Crohn's disease and ulcerative colitis. Eur Cytokine Netw. 2001;12:468-77.

42. Yamanishi $Y$, Karasuyama $\mathrm{H}$. Basophil-derived IL-4 plays versatile roles in immunity. Semin Immunopathol. 2016;38(5):615-22.

43. $\mathrm{Na} \mathrm{H}$, Cho M, Chung Y. Regulation of Th2 cell immunity by dendritic cells. Immune Netw. 2016;16:1-12.

44. Khokha R, Murthy A, Weiss A. Metalloproteinases and their natural inhibitors in inflammation and immunity. Nat Rev Immunol. 2013;13:649-65.

45. Abraham M, Shapiro S, Karni A, Weiner HL, Miller A. Gelatinases (MMP-2 and MMP-9) are preferentially expressed by Th1 vs. Th2 cells. J Neuroimmunol. 2005;163:157-64.

46. Goetzl EJ, Banda MJ, Leppert D. Matrix metalloproteinases in immunity. J Immunol. 1996;156:1-4.

47. Yong W, Krekoski CA, Forsyth PA, Bell R, Edwards DR. Matrix metalloproteinases and diseases of the CNS. Trends Neurosci. 1998;21:75-80.

48. Fields J, Dumaop W, Langford TD, Rockenstein E, Masliah E. Role of neurotrophic factor alterations in the neurodegenerative process in HIV associated neurocognitive disorders. J Neuroimmune Pharmacol. 2014:9:102-16.

49. Wu D. Neuroprotection in experimental stroke with targeted neurotrophins. NeuroRx. 2005;2:120-8.

50. Guillot-Sestier MV, Doty KR, Gate D, Rodriguez Jr J, Leung BP, Rezai-Zadeh K, Town T. II10 deficiency rebalances innate immunity to mitigate Alzheimerlike pathology. Neuron. 2015;85:534-48.

51. Chakrabarty P, Li A, Ceballos-Diaz C, Eddy JA, Funk CC, Moore B, DiNunno N Rosario AM, Cruz PE, Verbeeck C, et al. IL-10 alters immunoproteostasis in APP mice, increasing plaque burden and worsening cognitive behavior. Neuron. 2015;85:519-33.

52. Michaud JP, Rivest S. Anti-inflammatory signaling in microglia exacerbates Alzheimer's disease-related pathology. Neuron. 2015;85:450-2.

53. Wisniewski T, Drummond E. Developing therapeutic vaccines against Alzheimer's disease. Expert Rev Vaccines. 2016;15:401-15.

\section{Submit your next manuscript to BioMed Central and we will help you at every step:}

- We accept pre-submission inquiries

- Our selector tool helps you to find the most relevant journal

- We provide round the clock customer support

- Convenient online submission

- Thorough peer review

- Inclusion in PubMed and all major indexing services

- Maximum visibility for your research

Submit your manuscript at www.biomedcentral.com/submit
Biomed Central 